\title{
Can Full-Fat Dairy Intake Contribute to Preventing Diabetes and Cardiometabolic Issues?
}

\author{
Akbar Nikkhah* \\ Ferdows Pars Holding Co., Tehran, Iran \\ *Corresponding author: Akbar Nikkhah, Chief Highly Distinguished Professor and Scientist, Ferdows Pars Holding Co., Tehran, Iran; Email: anikkha@yahoo.com
}

Received: December 16, 2021; Accepted: December 20, 2021; Published: January 03, 2022

\begin{abstract}
This perspective article aimed to develop and at least partly address an important question if full-fat dairy intake can improve public health via contributing to diabetes and cardiometabolic issues prevention. Type 2 diabetes and associated complexities are related to lifestyle and nutritional-exercise regimens. Traditionally, the intake of low-fat or even fat-free dairy products has been suggested to reduce risks from metabolic complexities. This may be partly due to saturated fatty acids in full-fat dairy products that might theoretically cause cardiometabolic issues. However, the growing evidence suggests that full-fat vs. low-fat dairy have neutral or even beneficial effects on cardiometabolic health and diabetes prevention. Dairy products such as milk and yoghurt are considered functional foods that consist of various elements including vitamins, minerals, probiotics, anti-inflammatory, and bioactive molecules that work for body health in organized fashions. Hence, to sum up, full-fat dairy intake for diabetes and cardiometabolic issues prevention needs further profound assessment to enable optimizing the current public opinions.
\end{abstract}

Keywords: Full-fat dairy, Diabetes, Public health, Tradition, Science

\section{Introduction and Discussion}

This perspective article aimed to develop a question and discuss if full-fat dairy intake can contribute to preventing diabetes and cardiometabolic issues. Fresh milk and its products mainly yoghurt and cheese are considered health-promoting natural foods. However, from public perspective, full-fat dairy intake may be related to higher blood bad cholesterol and greater risks from diabetes and cardiometabolic issues [1,2]. As such, reduced-fat or even free-fat dairy intake has been recommended [3]. This position, however, has been challenged recently in various studies, suggesting neutral or even positive effects of full-fat dairy intake on glucose metabolism and cardiometabolic health $[4,5]$.

The correlation of dairy fat intake and cardiovascular diseases incidence has been weak [3]. It seems that individual saturated fatty acids (SFA) possess exclusive biochemical functions that might not be evaluated as a group. For example, myristic acid (14:0) is an activator of conversion of $\alpha$-linolenic acid to docosahexaenoic. It is also needed for the activation of proteins during biochemical pathways. Myristic acid and lauric acid (12:0) exhibit capacities to decrease blood bad cholesterol (LDL) [6]. Also, SFA particularly with shorter chains possess some potential to increase blood good cholesterol (HDL) and improve LDL/HDL ratio in yogurt eaters [4,7]. Thus, it has become possible to challenge the notion that SFA lead to increased blood cholesterol and cardiometabolic diseases. Realistically, a number of other effectors including social bond, nutrition, exercise, lifestyle, smoking, and stress are involved in the etiology of cardiometabolic diseases and cancer. Inflammation appears to an influential factor causing cardiovascular issues [7]. Dairy lipids such as phospholipids and sphingolipids may exhibit anti-inflammatory effects that can help prevent cardiovascular issues [7]. Furthermore, angiotensinconverting enzyme (ACE) inhibitors of milk protein may contribute to lowering blood pressure and its related heart coronary issues [8].

Notable, whole dairy products should be looked at as exclusive complexes because they possess unique functional roles [5]. Thinking of food matrices rather than single elements provides a more precise assessment of full-fat dairy products for diabetes and cardiometabolic diseases prevention [9]. Dairy nutrients, all in all, as a multipurpose complex can improve nutrient assimilation and function [7]. As such, decreasing the fat content of milk or yogurt may alter physiochemical structure and nature of dairy lipids. This may have unfavorable health outcomes.

In a large study with people from diverse countries and different continents, lower risk of mortality and cardiovascular issues were found in whole dairy consumers [10]. Moreover, full-fat dairy intake may have neutral or even beneficial impacts on cardiovascular health, with decreased risk of diabetes in yogurt eaters [11]. The odd-chain SFA are viewed as biomarkers of dairy fat intake and cardiometabolic diseases risks. Pentadecanoic acid (15:0), heptadecanoic acid (17:0) and transpalmitoleic acid (t16:1n-7) are some examples [12]. Measuring these biomarkers in older adults suggested an inverse relationship between dairy fat biomarkers with cardiovascular diseases and mortality [12]. Overall, it has become a challenging question if full-fat dairy intake can help prevent cardiometabolic diseases and diabetes or even obesity. Addressing this question will possess important implications for improving public health in the new times. 


\section{Implications}

Based on the recently enhanced understanding of the biological functions of dairy fats and other nutrients, seemingly, full-fat dairy intake may have no harmful effects on cardiovascular health. Instead, lifestyle, social bond, stress, and inflammation may be viewed as more important effectors. Favorable impacts of full-fat dairy intake on diabetes and related complexities may be seen in yogurt consumers. It is recommended that the worldwide public update/refine its understanding on full-fat dairy intake association with human wellbeing. This recommendation will have important implications for improving public health in the new era.

\section{Acknowledgment}

Nature is acknowledged for its inspirations towards innovative science perspectives.

\section{References}

1. Nikkhah A., B. Bekik (2012) Science and pseudo-science of milk implications for human health. In: Milk production, Nova Science Publisher, New York, USA, 15-20.

2. Turpeinen O (1979) Effects of cholesterol lowering diet on mortality from coronary disease and other causes. Circulation 59, 1-7. [crossref]

3. Lordan R, Tsoupras A, Mitra B, and Zebetakis I (2018) Dietary fats and cardiovascular disease: Do we really need to be concerned? Foods 7, 1-34.

4. Nikkhah A (2014) Yogurt the most natural and healthy probiotic: History Reveals. Journal of Probiotics and Health 1-2.

5. Hirahatake KM, Astrup A, Hill OJ, Slavin LJ, Allison BD, Maki CK (2020) Potential cardiometabolic health benefits of full-fat dairy: The evidence base: Advanced in Nutrition 11, 533-547. [crossref]

6. Nettleton JA, Legrand P, Mensink RP (2015) Issfal (2014) debate: It is time to update saturated fat recommendations. Annals of Nutrition and Metabolism. [crossref]

7. Lordan R and Zebetakis I (2017) The anti-inflammatory properties if dairy lipids. Journal of Dairy Science 100, 4197-4214.

8. Nikkhah A (2012) Milk for humans: Evolving perceptions of an all-time mother science. Russian Agricultural Sciences 38, 328-336.

9. Torning TK, Bertram HC, Bonjour Jp, De Groot L Dupont D, Feeney E, et al. (2017) Whole dairy matrix or single nutrients in assessment of health effects: current evidence and knowledge gaps. The American Journal of Clinical Nutrition 105, 10331045. [crossref]

10. Dehghan M, Mente A, Rangarajan S, Sheridan P, Mohan V, et al. (2018) Association of dairy intake with cardiovascular disease and mortality in 21 countries from five continents (PURE): a prospective cohort study. Lancet 392, 10161. [crossref]

11. Yu E and Hu FB (2018) Dairy products, dairy fatty acids and the prevention of cardiometabolic disease: A review of recent evidence. Current Atherosclerosis Reports 20, 24. [crossref]

12. de Oloveira Otto MC, Lemaitre RN, Song X, King IB, Sicovick DS, Mozaffarian D (2018) Serial measure of circulating biomarkers of dairy fat and total and causespecific mortality in older adults: the Cardiovascular Health Study. American Journal of Clinical Nutrition 108, 476-484. [crossref] 